\title{
Aplicación de la Integral Térmica como herramienta de control bioclimático y de modelamiento ambiental en el cultivo de tuna (Opuntia Ficus-indica)
}

\begin{abstract}
(c) (1) (2)(2)
Application of Thermal Integral as a tool for bioclimatic control and environmental modeling in the cultivation of prickly pear (Opuntia Ficusindica)
\end{abstract}

Hernán Chamorro Sevilla. ${ }^{1}$, Julia Desiree Velasteguí Cáceres. ${ }^{2}$

Recibido: 21-10-2020 / Revisado: 22-11-2020 /Aceptado: 12-12-2020/ Publicado: 02-01-2021

\begin{abstract}
.
DOI: https://doi.org/10.33262/concienciadigital.v4i1.1537

Introduction. In this work, the values of the daily accumulated grades of the experimental plot of prickly pear (Opuntia ficus-indica), installed in the Tunshi Experimental Center of the Higher Polytechnic School of Chimborazo (ESPOCH), are evaluated to improve prickly pear crops located in the Chingazo community of the Guano parish of the Guano Canton, Chimborazo Province, Ecuador. Objective. With the meteorological data acquired from the MERRA2 / GEOS 5.12.4 satellite, the phenological process of the experimental plot was carried out, calculating GDD, GDA and a projection of the Accumulated Degrees of Maturity (GMA). Methodology. To obtain the thermal summation, the mathematical model of the thermal integral was used, which allowed calculating the daily degree-days using satellite meteorological data; From which the daily maximum and minimum temperature data was used as input. Results. Approximate and referential data were obtained in the plot of the Tunshi experimental center of ESPOCH, with the indices of: $\mathrm{GDD}=9.1^{\circ} \mathrm{C}$; $\mathrm{GDA}=271^{\circ} \mathrm{C}$ in a month; GDA $=1,856.5^{\circ} \mathrm{C}$ in six months; GDA $=3,744.4^{\circ} \mathrm{C}$ in one year and GMA $=$ $9,357.3^{\circ} \mathrm{C}$ accumulated since July 26,2018 , when the experimental plot was replanted
\end{abstract}

1 Escuela Superior Politécnica de Chimborazo, Facultad de Recursos Naturales. Riobamba, Ecuador. hernan.chamorro@espoch.edu.ec, https://orcid.org/0000-0002-8531-7116

${ }^{2}$ Escuela Superior Politécnica de Chimborazo, Facultad de Recursos Naturales. Riobamba, Ecuador. julia.velastegui@espoch.edu.ec, https://orcid.org/0000-0002-6825-0853 
until December 31, 2020. Conclusion. The index of degrees accumulated both daily, monthly, semester and annually, show that the sector of the community of Chingazo is highly suitable since the prickly pear grows in minimum temperatures of $-16^{\circ} \mathrm{C}$ to 32 ${ }^{\circ} \mathrm{C}$ in hot places.

Keywords: Tuna (Opuntia ficus-índica); GDD; GDA; GMA; NASA Power Project; Environmental Modeling, Geographic Information Technologies.

\section{Resumen.}

Introducción. En este trabajo se evalúa los valores de los grados acumulados diarios de la parcela experimental de tuna (Opuntia ficus-índica), instalada en el Centro Experimental Tunshi de la Escuela Superior Politécnica de Chimborazo (ESPOCH), para mejorar en los cultivos de tuna ubicados en la comunidad Chingazo de la parroquia Guano del Cantón Guano, Provincia de Chimborazo, Ecuador. Objetivo. Con los datos metereológicos adquiridos del satélite MERRA2/GEOS 5.12.4 se realizó el proceso fenológico de la parcela experimental calculando de GDD, GDA y una proyección de los Grados de Madurez Acumulados (GMA). Metodología. Para obtener la sumatoria térmica, se utilizó el modelo matemático de la integral térmica que permitió calcular los Grados-día diarios utilizando los datos meteorológicos satelitales; del cual se utilizó como insumo los datos de temperatura máxima y mínima diaria. Resultados. Se obtuvieron datos aproximados y referenciales en la parcela del centro experimental Tunshi de la ESPOCH, de los índices de: $\mathrm{GDD}=9.1^{\circ} \mathrm{C}$; GDA $=271^{\circ} \mathrm{C}$ en un mes; $\mathrm{GDA}=1,856.5^{\circ} \mathrm{C}$ en seis meses; GDA $=3,744.4^{\circ} \mathrm{C}$ en un año y $\mathrm{GMA}=9,357 \cdot 3^{\circ} \mathrm{C}$ acumulados desde el 26 de julio del 2018 que fue replantada la parcela experimental hasta el 31 de diciembre del 2020. Conclusión. El índice de los grados acumulados tanto diarios, mensuales, semestrales y anuales, muestran que el sector de la comunidad de Chingazo es altamente apto ya que la tuna se desarrolla en temperaturas mínimas de hasta $-16^{\circ} \mathrm{C}$ hasta $32^{\circ} \mathrm{C}$ en lugares calientes.

Palabras claves: Tuna (Opuntia ficus-índica); GDD; GDA; GMA; NASA Power Project, Modelación Ambiental, Tecnologías de Información Geográfica.

\section{Introducción.}

El cultivo de la tuna, es una de las actividades que se viene desarrollando desde épocas prehispánicas (hace 8000 años; Bravo 1991), convirtiéndose en los lugares en donde se desarrola en el de mayor importància alimenticia por sus diverses utilidades. Es llamada tuna (Opuntia ficus-indica) a la planta y al fruto (Microenprendimientos, s/f).

A la llegada de los españoles al mar Caribe a lo que hoy se conoce como Haití y República Dominicana, los nativos les mostraron unos frutos de color rojo de tuna que se deriva de la palabra tun en la lengua nativa. Desde entonces los conquistadores comenzaron a consumir 
los frutos de nopal. El primer autor que descubrió es Oviedo y Valdés quien describió el fruto y la planta (FAO, 2018).

En la actualidad los nopales son parte del entorno natural y de los sistemas agrícolas y son consideradas las cactáceas de mayor valor económico en el mundo. Es cultivada en América, África, Asia, Europa y Oceanía. En el caso de América se encuentra desde Canadá hasta la Patagonia, Argentina desde el nivel del mar hasta los 5100 metros de altitud como es el caso del Perú (FAO, 2018).

La tuna en el Ecuador es cultivada cuatro variedades que son la tuna amarilla sin espina, la amarilla con espina, la blanca y la silvestre, con mayor arraigo en las provincias de Imbabura, Loja, Santa elena y Tungurahua, aproximadamente en unas 180 hectáreas (MAG, 2017).

En el cantón Guano, en la provincia de Chimborazo, se cultiva la tuna blanca y amarilla, aunque en menor proporción y que es distribuida localmente, alrededor de 600 agricultores agrupados en la Asociación de Granjero Guaneño que adquieren la tuna para procesar y transformar en tónicos medicinales, mermeladas, champús y vinos en su propia planta procesadora (Maggi, 2017).

La tuna se considera una planta arbustiva prerenne que alcanza una altura de 5 metros de altura y de 3 a 4 metros de diámetro de copa. Tiene una raíz fibrosa, cilíndrica y de rápido crecimiento, su tallo es muy ramificado. Las pencas pueden tener un color verde claro o verde gris, su fruto de forma redonda de consistencia carnosa y jugosa de pulpa dulce aromática con textura firme, arenosa y su color varía dependiendo del tipo (Salas, 2020).

Ésta planta cumple con todos los requerimientos para ser considerada un cultivo energético, por tener un mecanismo fotosintético en la cual las plantas abren sus estomas para captar el $\mathrm{CO}_{2}$ en la noche conocido como metabolismo del ácido crassuláceo (CAM); esto es cuando las temperaturas son bajas y la humedad es más alta del dia, esto reduce la pérdida de agua y ofrece más posibilidades de producción de biomasa (Falasca, Bernabé y Lamas, 2011).

Las mejores condiciones para el cultivo de tuna son las zonas áridas con temperatura promedio de 16 a $26 \mathrm{C}$, tener una alta luminosidad y una hunedad relativa de 55 a $85 \%$. La altitud más adecuada es de 800 a 2,500 msnm. Las características del suelo donde se desarrollan son arenosos-calcáreos, sueltos, fértiles, poco profundos y con un $\mathrm{pH}$ poco alcalino (Salas, 2020).

Sin embargo, las bajas temperaturas afectan al cultivo, por lo que su tolerancia a temperaturas mínimas está en el orden de $0^{\circ}$ a $10^{\circ} \mathrm{C}$, aunque ciertas variedades pueden soportar temperaturas bajas de hasta $16^{\circ} \mathrm{C}$ bajo cero (Ecured, 2018). 
Como se conoce la fenología de las plantas depende de la temperatura, insolación y humedad en todos sus fenómenos biológicos que son indispensables conocer e identificar para la toma de decisiones, empezando por la brotación de yemas, inflorescencia y la maduración de los frutos (Amaya, 2014).

Para identificar las accesiones y variedades es el color del fruto, tanto interno como externo. Aunque el color del fruto es dinámico y cambiante con la etapa de la madurez, en tanto que el color de pulpa cambia también con la madurez, por lo que la exploración debe ser guiada por un experto (FAO, 2018).

El período de desarrollo de la flor, desde el brote hasta la madurez es de aproximadamente de 25 a 37 dias. Mientras que el desarrollo varía según las condiciones climáticas en especial con la temperatura desde 59 a 150 días (Brustch 1979, Nerd et al. 1990, Pimienta-Barrios 1990, Inglese \& Barbera 1992, Kuti 1992, Nieddu \& Spano 1992) (Microenprendimientos, s/f).

Por otro lado, las características de la atmósfera y los factores influyentes en el clima y los fenómenos atmosféricos, tienen gran importancia una vez detectados por el satélite para su posterior análisis, mejorando los mapas del tiempo aproximadamente desde los años 60 (Izquierdo y Pueyo, 1991).

Los satélites meteorológicos, pueden observar áreas de los continentes y de los océanos de los cuales se tiene poca información, obteniendo datos sobre nubes, temperatura y humedad del aire, viento, temperatura del suelo y del mar, corrientes marinas, inestabilidad atmosférica y precipitaciones que son elementos asociados con el pronóstico del tiempo (Renom, 2011). La información meteorológica, permite la localización y seguimiento de ciclones tropicales o de tormentas, evaluación de áreas con probabilidad de inundaciones o áreas inundadas, determinar áreas con riesgo de incendios, evaluación de cosechas, contaminación del aire o agua o temas relacionados (Renom, 2011).

La Nasa Power es un proyecto de las ciencias aplicadas, que proporciona información solar y meteorológica del mundo para apoyar la energía renovable, la eficiencia energética y las necesidades agrícolas, a través de su programa de investigación en Ciencias de la Tierra proporciona datos importantes para el estudio del clima y procesos climáticos. Los mismos que son estimaciones climatológicas promedio a largo plazo (NASA, 2019).

Además, los valores promedios diarios lo suficientemente precisos tanto meteorológicos como solares en formato de series de tiempo, basados en modelos y satélites en regiones donde las mediciones de superficie son escasos o inexistentes (NASA, 2019). 
Uno de los modelos matemáticos que se utiliza para determinar los grados de temperatura acumulados diarios por cada planta es la integral térmica que es un parámetro importante para la definición de las estrategias de diseño, los requerimientos de climatización ya sea natural o artificial. Básicamente los Grados-día Diarios (GDD), no es otra cosa que la diferencia entre la temperatura base de referencia y la temperatura media diaria exterior (Fuentes-Freixanet, 2010).

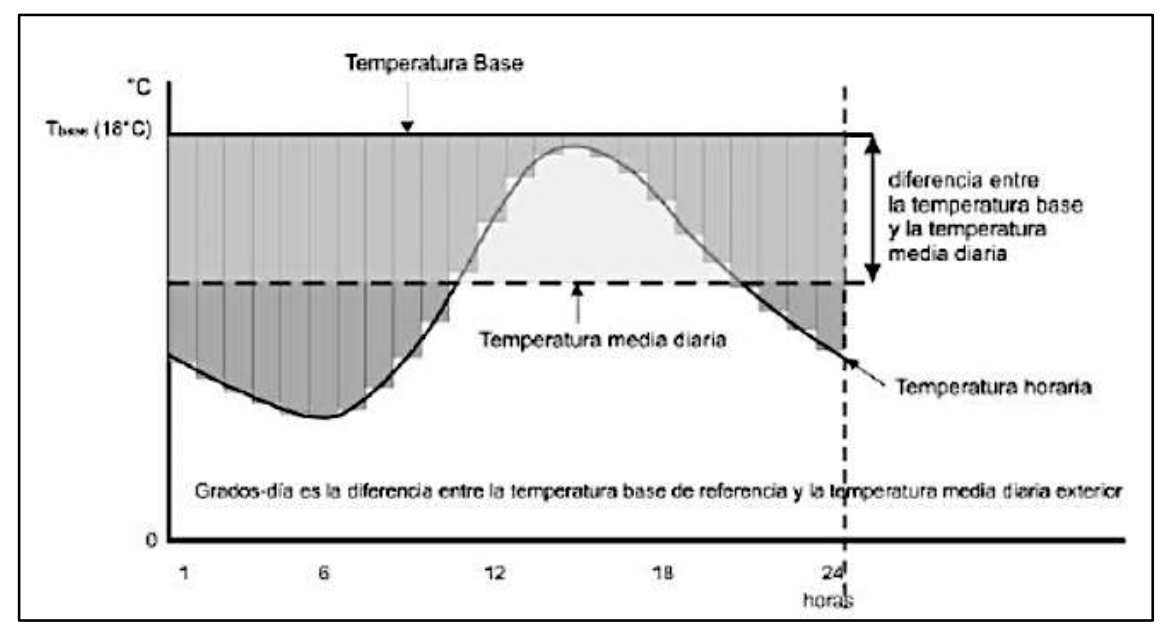

Figura 1. Esquema de la definición Grado-dia Fuente: Fuentes-Freixanet (2010).

Los Grados-hora Diarios (GHD) son las diferencias acumuladas de la temperatura hora a hora y a lo largo de todo el día; pero si estos datos se dividen para 24 horas se obtendrán los GDD. Ver Figura 1.

Fuentes-Freixanet (2010), define que los Grados-día son los requerimientos acumulados en cierto período de tiempo (mensual, semanal, diario u horario) de calentamiento o enfriamiento necesarios para alcanzar la zona optima. Si se habla de GDD específicamente es e déficit o superávit acumulado de los 365 días del año y viene dada por la fórmula:

$$
\begin{aligned}
& \qquad G D=\sum_{1}^{365}\left(T-T_{\text {base }}\right) \\
& \text { donde: } \\
& \text { GD }=\text { Grados-Día }\left({ }^{\circ} \mathrm{C}\right) \\
& \mathrm{T}_{\text {base }}=\text { Temperatura base (límite de referencia, inferior o superior de } \\
& \text { confort }\left({ }^{\circ} \mathrm{C}\right) \\
& \mathrm{T}=\text { Temperatura media diaria }\left({ }^{\circ} \mathrm{C}\right)
\end{aligned}
$$

Por la variación diaria de las temperaturas horarias, generalmente, de manera simplificada se emplean los Grados-día mensuales; esto es las diferencias entre la temperatura base de 
referencia y la temperatura media diaria acumulada durante el mes, con la siguiente ecuación: (Fuentes-Freixanet, 2010).

$$
G D=\sum_{1}^{n}\left(T-T_{\text {base }}\right)
$$

donde:

$\mathrm{n}=$ número de días del mes

Si no se tiene la información diaria se puede usar los datos mensuales de temperatura multiplicados por el número de días del mes, que representan los datos promedio de todos los días del mes (Fuentes-Freixanet, 2010).

$$
G D=n \cdot\left(T_{\text {mensual }}-T_{\text {base }}\right)
$$

Para realizar un análisis de mayor precisión, se puede hacer de manera horaria en Gradoshora; esto es de manera acumulada anual (de 1 a 8,760 horas) o mensual (de 1 a 24 horas), para lo cual se utiliza la fórmula: (Fuentes-Freixanet, 2010).

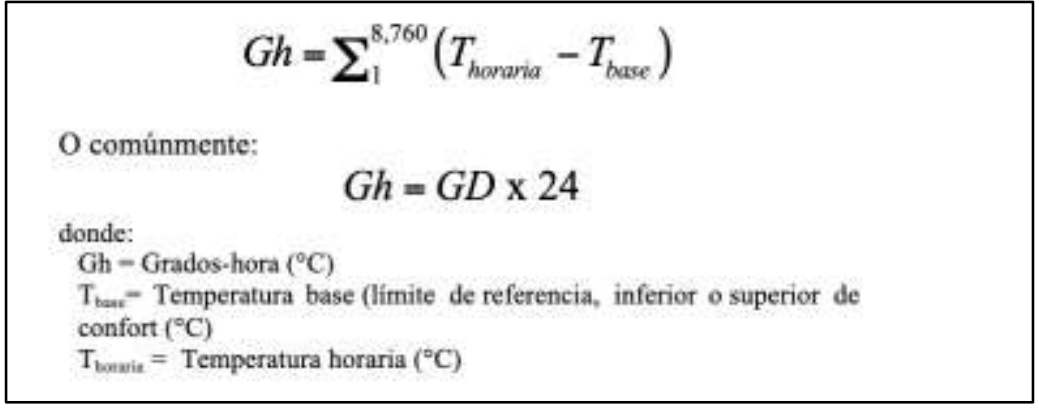

Los Grados Días Acumulados (GDA) se obtiene de la suma entre la temperatura máxima y temperatura mínima, dividiendo para 2 y cuyo resultado se resta la temperatura base. Como muestra la formula a continuación (Velásquez, Rosales, Rodríguez y Salas, 2015).

$$
G D A=\sum_{1}^{n}\left(\frac{T_{\max }+T_{\min }}{2}\right)-T_{b}
$$

donde:

$$
\begin{aligned}
& T_{\max }=\text { Temperatura Máxima } \\
& T_{\min }=\text { Temperatura Mínima } \\
& T_{b}=\text { Temperatura base }
\end{aligned}
$$


Los Grados de Madurez Acumuladas (GMA), es el índice más usado para la cosecha de frutos, pero debe diferenciarse la madurez fisiológica de la madurez comercial. La primera es aquella que se alcanza luego que se ha completado el desarrollo mientras que la segunda se refiere al estado en el cual es requerido por el mercado (López, 2003).

El GMA se obtuvo aplicando la Integral Térmica y es la suma de los GDA desde la fecha de la siembra hasta llegar al valor de la madurez fisiológica del cultivo, y se calcula con la fórmula:

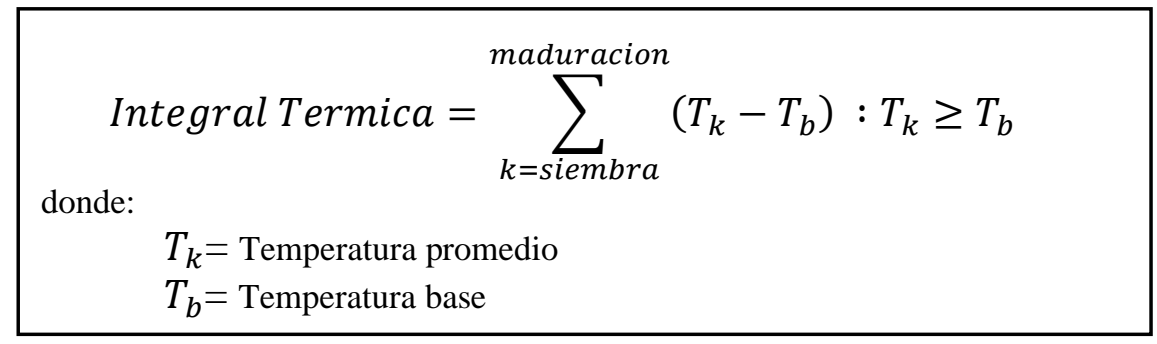

La poca información técnica disponible sobre los datos fenológicos de la tuna en el sector de Chingazo del cantón Guano de la provincia de Chimborazo, son las razones que sustentan el desarrollo de la presente investigación, razón por la cual se plantearon como objetivos en primer lugar la necesidad de adquirir los datos metereológicos satelitales y en segundo lugar calcular el proceso fenológico de la parcela experimental, el cálculo de GDD, GDA, la proyección de los Grados de Madurez Acumulados (GMA) y la generación de estrategias de manejo técnico que orienten la etapa fenológica de la tuna en el sector antes mencionado.

\section{Metodologia.}

En este estudio se analiza el comportamiento diario en el crecimiento de la tuna en el sector de "Chingazo", situado en la parroquia Guano del cantón Guano, cuyas coordenadas cubre el área ubicada a 1 ${ }^{\circ} 37^{\prime} 10^{\prime \prime} \mathrm{S} 78^{\circ} 35^{\prime} 14^{\prime \prime} \mathrm{O}, 2666$ metros sobre el nivel del mar. cerca de la Ciudad de Riobamba se encuentra la parcela en la comunidad Chingazo del cantón Guano de la provincia de Chimborazo, como muestra la Figura 2. a partir de los datos meteorológicos de la temperatura que se obtuvo del proyecto Power de la Administración Nacional de Aeronáutica y el Espacio (NASA, por sus siglas en inglés) (https://power.larc.nasa.gov/dataaccess-viewer/ ), dicha información se encuentra en formato de archivo separado por comas (csv), que fue importado a la hoja electrónica para realizar los cálculos de los índices del GDD, GDA y GMA; aplicando el método matemático de la integral térmica y utilizando las temperaturas máxima y mínima diarias registradas de la zona de interés desde el 26 de julio del 2018, fecha en la que se trasplantó en la parcela experimental en el Centro Experimental Tunshi de la ESPOCH. 


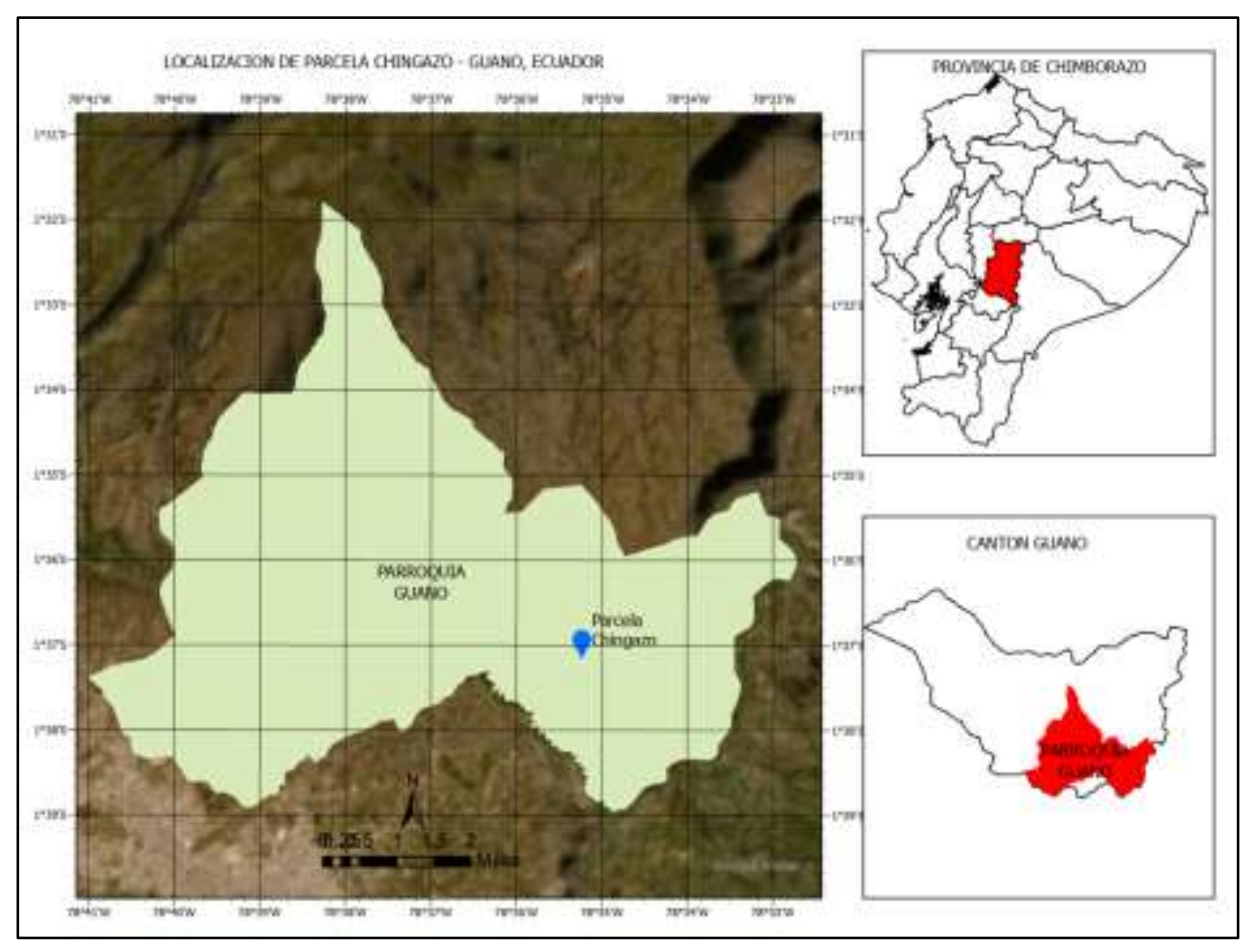

Figura 2. Ubicación de la comunidad Chingazo

Fuente: Elaboración propia

La siembra de la parcela experimental en el Centro Experimental Tunshi, como se indicó antes, se realizó el 26 de julio del 2018 con plantas adquiridas, tratadas y codificadas antes del trasplante, de la cual se sacaron los datos para este estudio. Como muestra la figura 3.

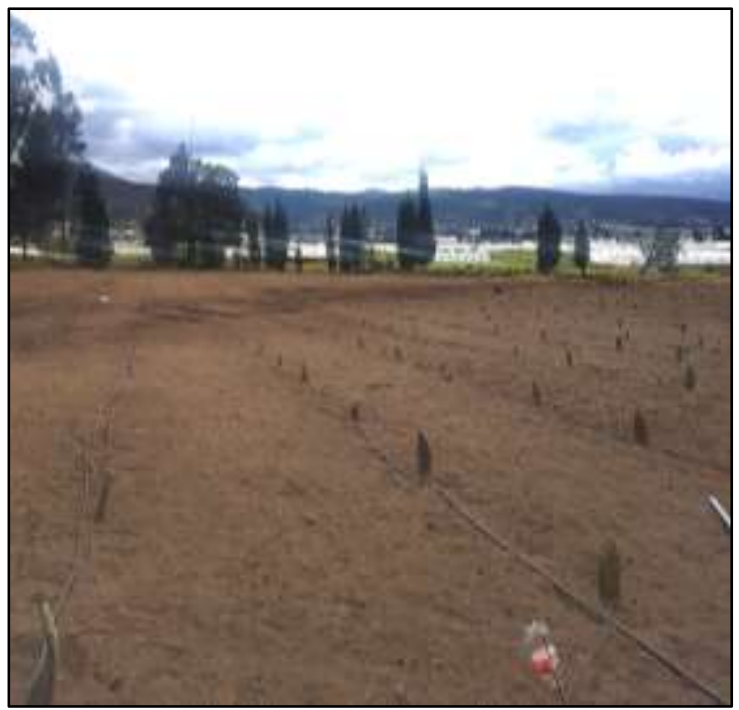

Figura 3. Trasplante de plantas madre en el Centro Experimental Tunshi Fuente: Elaboración propia 
Los GDD o sumatoria térmica fue calculada por el método de Arnold (1960), en el cual los datos de la temperatura fueron extraídos del satélite MERRA2/GEOS 5.12.4 que pertenecen al proyecto Power de la NASA y que proporcionan datos de temperaturas, radiación solar y precipitación.

Para el cálculo de los GDD mensuales se tomó la temperatura máxima (T2M_MAX) y la temperatura mínima (T2M_MIN) registradas en el área de estudio, como primer paso se procedió a sacar el promedio de las 2 temperaturas representadas en la fórmula como $\left(T_{k}\right)$ a cuyo resultado se le resta la temperatura base $\left(T_{b}\right)$, finalmente, se suma los valores de 30 días. Como muestra la formula:

$$
G D D=\sum_{1}^{30}\left(T_{k}-T_{b}\right)
$$

Este resultado corresponde a los grados del primer mes acumulados por la planta de tuna, este cálculo se realizó mes a mes durante el período establecido para esta investigación. Para los valores de GDA se tomaron los valores acumulados del GDD para establecer la madurez fisiológica tomando en consideración la fecha inicial el trasplante y como fecha final el día 201 que corresponde a julio del 2019, utilizando la formula:

$$
G D A=\sum_{1}^{365}\left(\frac{T_{\max }+T_{\min }}{2}\right)-T_{b}
$$

Una vez procesados los datos y con la ayuda de una hoja electrónica, y aplicando las formulas mencionadas anteriormente para obtener los índices de GDD y GDA que determinaron los grados acumulados de la tuna en la parcela, se procedió a sumar los grados acumulados hasta el 31 de diciembre dl 2020. Posteriormente, se analizó la acumulación de grados de la planta en las condiciones climáticas de la zona en la que se encuentra la parcela experimental.

\section{Resultados.}

Para la siembra de las plantas madres de tuna, se adquirieron 1,500 plantas de tunas de las tres variedades (blanca, amarilla y roja), las mismas que fueron trasplantadas el 26 de julio del 2018, a una distancia de siembra de 2 metros por fila y 2 metros por hilera.

Antes se ser trasplantadas los cladodios (orejas) fueron desinfectados con fungicida (caldo bordelés) más aceite agrícola.

Se realizó una codificación de las plantas, las mismas que se encuentran detalladas en un plano, cada planta posee un código, por ejemplo 3.25, lo que significa que la planta pertenece a la fila tres y la planta número 25; es decir antes del punto me indica el número de fila y 
luego del punto el número de planta. La codificación anteriormente detalla permitió ingresar a una base de datos iniciales tanto fenológicos como biométricos durante la etapa de investigación. En la tabla 2. Se encuentra los cálculos realizados del promedio de las temperaturas, la temperatura base (T_BASE) que corresponde a la temperatura más baja registrada en la zona y el cálculo del GDD.

\begin{tabular}{llrrrrrrr}
\hline LAT & LON & YEAR & DOY & T2M_MAX & T2M_MIN & PROM & T BASE & GDD \\
\hline-175.039 & -7.862 .849 & 2018 & 206 & 15,14 & 6,23 & 10,7 & 1,56 & 9,1 \\
-175.039 & -7.862 .849 & 2018 & 207 & 15,9 & 6,34 & 11,1 & 1,56 & 9,6 \\
-175.039 & -7.862 .849 & 2018 & 208 & 15,46 & 6,28 & 10,9 & 1,56 & 9,3 \\
-175.039 & -7.862 .849 & 2018 & 209 & 16,12 & 4,79 & 10,5 & 1,56 & 8,9 \\
-175.039 & -7.862 .849 & 2018 & 210 & 15,75 & 4,43 & 10,1 & 1,56 & 8,5 \\
-175.039 & -7.862 .849 & 2018 & 211 & 16,25 & 6,27 & 11,3 & 1,56 & 9,7 \\
-175.039 & -7.862 .849 & 2018 & 212 & 13,91 & 5,28 & 9,6 & 1,56 & 8,0 \\
-175.039 & -7.862 .849 & 2018 & 213 & 15,27 & 6,55 & 10,9 & 1,56 & 9,4 \\
-175.039 & -7.862 .849 & 2018 & 214 & 15,95 & 6,84 & 11,4 & 1,56 & 9,8 \\
-175.039 & -7.862 .849 & 2018 & 215 & 16,16 & 3,48 & 9,8 & 1,56 & 8,3 \\
-175.039 & -7.862 .849 & 2018 & 216 & 14,95 & 5,43 & 10,2 & 1,56 & 8,6 \\
-175.039 & -7.862 .849 & 2018 & 217 & 17 & 5,72 & 11,4 & 1,56 & 9,8 \\
-175.039 & -7.862 .849 & 2018 & 218 & 17,17 & 6,36 & 11,8 & 1,56 & 10,2 \\
-175.039 & -7.862 .849 & 2018 & 219 & 16,48 & 7,48 & 12,0 & 1,56 & 10,4 \\
-175.039 & -7.862 .849 & 2018 & 220 & 15,92 & 7,17 & 11,5 & 1,56 & 10,0 \\
-175.039 & -7.862 .849 & 2018 & 221 & 13,95 & 6,29 & 10,1 & 1,56 & 8,6 \\
-175.039 & -7.862 .849 & 2018 & 222 & 12,73 & 4,25 & 8,5 & 1,56 & 6,9 \\
-175.039 & -7.862 .849 & 2018 & 223 & 15,34 & 4,85 & 10,1 & 1,56 & 8,5 \\
-175.039 & -7.862 .849 & 2018 & 224 & 15,09 & 5,1 & 10,1 & 1,56 & 8,5 \\
-175.039 & -7.862 .849 & 2018 & 225 & 16,29 & 4,35 & 10,3 & 1,56 & 8,8 \\
-175.039 & -7.862 .849 & 2018 & 226 & 15,76 & 4,09 & 9,9 & 1,56 & 8,4 \\
-175.039 & -7.862 .849 & 2018 & 227 & 15,95 & 5,37 & 10,7 & 1,56 & 9,1 \\
-175.039 & -7.862 .849 & 2018 & 228 & 15,27 & 6,3 & 10,8 & 1,56 & 9,2 \\
-175.039 & -7.862 .849 & 2018 & 229 & 14,74 & 6,13 & 10,4 & 1,56 & 8,9 \\
-175.039 & -7.862 .849 & 2018 & 230 & 16,69 & 5,81 & 11,3 & 1,56 & 9,7 \\
-175.039 & -7.862 .849 & 2018 & 231 & 16,82 & 3,42 & 10,1 & 1,56 & 8,6 \\
-175.039 & -7.862 .849 & 2018 & 232 & 15,75 & 4,78 & 10,3 & 1,56 & 8,7 \\
-175.039 & -7.862 .849 & 2018 & 233 & 15,8 & 6,46 & 11,1 & 1,56 & 9,6 \\
-175.039 & -7.862 .849 & 2018 & 234 & 15 & 5,74 & 10,4 & 1,56 & 8,8 \\
-175.039 & -7.862 .849 & 2018 & 235 & 16,76 & 4,75 & 10,8 & 1,56 & 9,2 \\
\hline & & & & & & & & \\
- & & & & & \\
$-1,56$ &
\end{tabular}

Tabla 1. Muestra del Cálculo del GDD Fuente: Elaboración propia 
Aplicando la fórmula a los datos de la tabla 1. Se obtiene el siguiente resultado:

$$
G D=\sum_{1}^{30}\left(T_{k}-T_{b}\right)=271.1^{\circ} \mathrm{C}
$$

El valor de $271.1^{\circ} \mathrm{C}$ corresponde al primer mes acumulado por la planta de tuna, este cálculo se puede realizar mes a mes para llevar un control de los grados acumulados. A continuación, en la tabla 1, se muestra un resumen de los cálculos diario, mensual y anual de este índice.

\begin{tabular}{lllll}
\hline GDA & Diario & Mensual & semestral & Anual \\
\hline Valor & 9.1 & 271.1 & $1,856.5$ & $3,744.4$ \\
\hline
\end{tabular}

Tabla 1. Valores de GDD

Fuente: Elaboración propia

Una vez procesados los datos y con la ayuda de una hoja electrónica, y aplicando las formulas mencionadas anteriormente para obtener los índices de GDD y GDA que determinaron los grados acumulados de la tuna en la parcela, se procedió a sumar los grados acumulados hasta el 31 de diciembre dl 2020, dando como resultado un valor de 9,357.3 ${ }^{\circ} \mathrm{C}$. Posteriormente, se analizó el crecimiento de la planta en las condiciones climáticas de la zona en la que se encuentra la parcela experimental; esto permite tomar decisiones en implementar un sistema de riego.

Para los valores de GDA se tomaron los valores acumulados del GDD para establecer la madurez fisiológica tomando en consideración la fecha inicial el trasplante y como fecha final el día 201 que corresponde a julio del 2019, utilizando la formula:

$$
G D A=\sum_{1}^{365}\left(\frac{T_{\max }+T_{\min }}{2}\right)-T_{b}=3,744.4^{\circ} \mathrm{C}
$$

Esto implica que la tuna durante un año con las temperaturas máximas y mínimas registradas en la zona ha acumulado $3,744.4^{\circ} \mathrm{C}$.

En este caso el $\left(\frac{T_{\max }+T_{\min }}{2}\right)$ es promedio de las temperaturas máxima y mínima y el $T_{b}$ es la temperatura base; por lo tanto, el GDA es el valor en grados de temperatura que acumula diariamente la planta de tuna. A continuación, se visualiza en la tabla 2. un ejemplo de los valores de grados diarios acumulados. 


\begin{tabular}{crr}
\hline GDD & T BASE & \multicolumn{2}{c}{ GDA } \\
\hline 10,7 & 1,56 & 9,1 \\
11,1 & 1,56 & 9,6 \\
10,9 & 1,56 & 9,3 \\
10,5 & 1,56 & 8,9 \\
10,1 & 1,56 & 8,5 \\
11,3 & 1,56 & 9,7 \\
9,6 & 1,56 & 8,0 \\
10,9 & 1,56 & 9,4 \\
11,4 & 1,56 & 9,8 \\
9,8 & 1,56 & 8,3 \\
10,2 & 1,56 & 8,6 \\
11,4 & 1,56 & 9,8 \\
11,8 & 1,56 & 10,2 \\
12,0 & 1,56 & 10,4 \\
11,5 & 1,56 & 10,0 \\
10,1 & 1,56 & 8,6 \\
8,5 & 1,56 & 6,9 \\
10,1 & 1,56 & 8,5 \\
10,1 & 1,56 & 8,5 \\
10,3 & 1,56 & 8,8 \\
\hline
\end{tabular}

Tabla 2. Muestra del cálculo de GDA

Fuente: Elaboración propia.

El GMA acumulado desde el 26 de julio de 2018 hasta el 31 de diciembre de 2020 se obtiene sumando los valores de GDA, dando el valor de $9,357.3^{\circ} \mathrm{C}$.

\section{Conclusiones.}

- Los valores obtenidos en esta investigación son referenciales y aproximados porque dependen de la temperatura captada por el satélite, pero que pueden ayudar en el incremento de la producción de tuna de los agricultores del sector de Chingazo, implementando un sistema de riego de agua por goteo.

- El índice de los grados acumulados tanto diarios, mensuales, semestrales y anuales, muestran que el sector de la comunidad de Chingazo es altamente apta ya que la tuna se desarrolla en temperaturas mínimas de hasta $-16^{\circ} \mathrm{C}$ hasta $32^{\circ} \mathrm{C}$ en lugares calientes.

- La variedad cultivada en la zona es adaptable a temperaturas de hasta $0^{\circ} \mathrm{C}$ hasta los $26^{\circ} \mathrm{C}$ y en la temperatura mínima registrada por el satélite en la zona es de $1.56^{\circ} \mathrm{C}$ lo cual implica que durante todo el ciclo investigado la tuna ha adquirido Grados-día.

- Para obtener valores más exactos de los Grados-día y Grados-día acumulados de la tuna, es recomendable utilizar datos recolectados por alguna estación metereológica 
terrestre cercana. Las 1,500 muestras han sido cultivadas en una parcela de en un área de 0.8 hectáreas para realizar un seguimiento minucioso del crecimiento y los Grados Diarios Acumulados en función del tiempo.

- Desde el 26 de julio del 2018 hasta la fecha actual, en la parcela experimental Tunshi de la ESPOCH han crecido las hojas de la tuna, pero aún no se tiene brote de frutos. Es importante mencionar también que está implementado un sistema de riego de agua por goteo.

\section{Referencias bibliográficas.}

Administración Nacional de Aeronáutica y el Espacio - NASA, (2019). Power Data Metodology. Recuperado desde: https://power.larc.nasa.gov/docs/methodology/

Amaya, J. (2014). El cultivo de tuna (Opuntia ficus índica). Recuperado desde: https://es2.slideshare.net/pedrogutierrez758399/manual-tecnico-de-tuna

Arnold, C. 1960. Maximun-minimum temperatures as a basis for computing heat units. Journal of the American Society for Horticultural Sciences, Alexandria 76:682-692.

Domínguez, J. A. Z., Covarrubias, J. M., Zegbe-Domínguez, J. A., \& Mena-Covarrubias, J. Modificación de la floración, maduración y época de cosecha del nopal tunero (Opuntia spp.).

Ecured.

Nopal.

Recuperado

desde:

https://www.ecured.cu/Nopal\#: :text=El\%20nopal\%20se\%20desarrolla\%20bien,ata que $\% 20 \mathrm{de} \% 20$ plagas\%20y\%20enfermedades. https://www.ecured.cu/index.php?title=Nopal\&oldid=3038011

Falasca, S., Bernabé, M. A., \& Lamas, C. (2011). Aptitud agroclimática de áreas áridas y semiáridas de Argentina para el cultivo de tuna (Opuntia ficus indica) como fuente de bioetanol. Quebracho-Revista de Ciencias Forestales, 19(1-2), 66-74. Recuperado desde: https://www.redalyc.org/pdf/481/48122207007.pdf

Fuentes-Freixanet, V. (2010). Grados-Día como herramienta de control bioclimático. Recuperado desde: https://www.researchgate.net/publication/280949538_GradosDia_como_herramienta_de_control_bioclimatico

Izquierdo, L., Pueyo, L. (1991). Satélites Metereológicos. Recuperado de: https://dialnet.unirioja.es/descarga/articulo/2773793.pdf

López, A. (2003). Manual Para la Preparación y Venta de Frutas y Hortalizas. Recuperado desde: http://www.fao.org/3/Y4893S/y4893s04.htm 
Maggi, E. (2017). La tuna genera recursos a 600 campesinos de Guano. Recuperado de: https://www.eltelegrafo.com.ec/noticias/regional/1/la-tuna-genera-recursos-a-600campesinos-de-guano

MAG-Ministerio Agricultura y Ganadería. (2017). La producción de tuna, una actividad rentable. Recuperado de: https://www.agricultura.gob.ec/la-produccion-de-tuna-unaactividad-rentable/

Microemprendimientos. $\quad(\mathrm{s} / \mathrm{f})$. $\quad$ recuperado http://www.agrobit.com/Documentos/I_1_7_Cultivos/339_mi000001tu[1].htm

Renom, M. (2011). Satélites metereológicos. Recuperado de: http://meteo.fisica.edu.uy/Materias/TICA/SatMeteoapuntes.pdf

Organización de las Naciones Unidas para la Alimentación y la Agricultura (FAO, por sus siglas en Inglés). (2018). Ecología del cultivo, manejo y usos del nopal. pp. 2-12. Recuperado de: http://www.fao.org/3/i7628es/I7628ES.pdf

Salas, D. (2020). Proyectos peruanos - Cultivo de tuna. Recuperado desde: https://proyectosperuanos.com/siembra_y_cultivo_de_tunas/

Velázquez, J., Rosales, A., Rodríguez, H., \& Salas, R. (2015). Determinación de las etapas de inicio de macollamiento, inicio de primordio, floración y madurez en la planta de arroz,con el sistema s, v y r correlacionado con la sumatoria térmica. Agronomía Costarricense, 39(2), 121-130. Recuperado desde: http://www.scielo.sa.cr/scielo.php?script=sci_arttext\&pid=S037794242015000200121\&lng=en\&tlng=es.

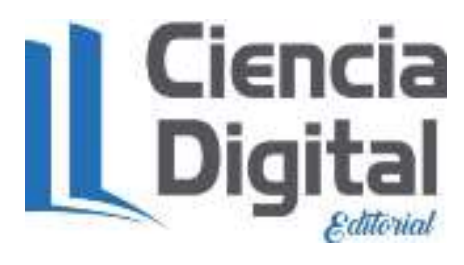




\section{PARA CITAR EL ARTÍCULO INDEXADO.}

Chamorro Sevilla, H., \& Velasteguí Cáceres, J. D. (2021). Aplicación de la Integral Térmica como herramienta de control bioclimático y de modelamiento ambiental en el cultivo de tuna (Opuntia Ficus-indica). ConcienciaDigital, 4(1), 106-120. https://doi.org/10.33262/concienciadigital.v4i1.1537

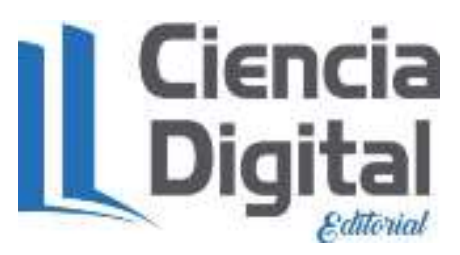

El artículo que se publica es de exclusiva responsabilidad de los autores y no necesariamente refleja el pensamiento de la Revista Conciencia Digital.

El artículo queda en propiedad de la revista y, por tanto, su publicación parcial y/o total en otro medio tiene que ser autorizado por el director de la Revista Conciencia Digital.

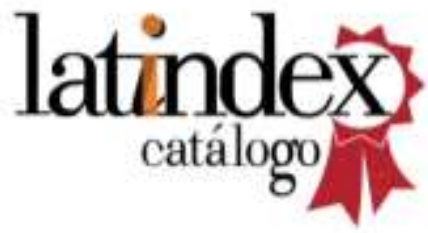

Conciencia

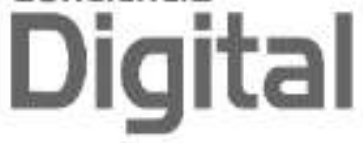

